\title{
Retraction Note to: Lupeol inhibits migration and invasion of colorectal cancer cells by suppressing RhoA-ROCK1 signaling pathway
}

\author{
Yiwen Jiang $^{1} \cdot$ Dan Hong ${ }^{1} \cdot$ Zhefeng Lou $^{1} \cdot$ Xuezi Tu${ }^{1} \cdot$ Longjin Jin $^{1}$
}

Published online: 16 July 2021

๑) Springer-Verlag GmbH Germany, part of Springer Nature 2021

Retraction Note to: Naunyn-Schmiedeberg's Archives of Pharmacology (2020) 393:2185-2196 https://doi.org/10.1007/s00210-020-01815-3

The Editor-in-Chief has retracted this article because of significant concerns regarding a number of the figures, specifically the Western blots presented in Figures 4B and 5A, and the confocal microscopy analysis presented in Figure 3B. The authors did not respond to the request for raw data and the Editor was also unable to contact authors' institution. The Editor-in-Chief therefore no longer has confidence in the integrity of the data in this article.
None of the authors responded to the correspondence from the Editor or Publisher about this retraction.

Publisher's note Springer Nature remains neutral with regard to jurisdictional claims in published maps and institutional affiliations.

The original article can be found online at https://doi.org/10.1007/ s00210-020-01815-3.

Longjin Jin

jlj@wmu.edu.cn

1 School of Laboratory Medicine and Life Science, Wenzhou Medical University, Wenzhou 325000, China 